\title{
Las actitudes prejuiciosas del alumnado uni- versitario hacia los inmigrantes en situación irregular: un estudio exploratorio
}

\section{Adrián Segura-Robles ${ }^{1}$, Inmaculada Alemany-Arrebola ${ }^{2}$, Miguel Ángel Gallardo-Vigil ${ }^{3}$}

${ }^{1}$ Departamento de Didáctica y Organización Escolar, Facultad de Ciencias de la Educación de Huelva, Huelva.

${ }^{2}$ Departamente de Psicología evolutiva y de la Educación, Facultad de Educación y Humanidades de Melilla, Melilla.

${ }^{3}$ Departamento de Métodos de Investigación y Diagnóstico en Educación, Facultad de Educación y Humanidades de Melilla, Melilla.

\section{España}

Correspondencia: Adrián Segura Robles. Paseo marítimo edif. Playa $1 \mathrm{n}^{\circ} 282^{\circ} \mathrm{C}, 52006$, Melilla, Melilla (España.). E-mail: adriansegurarobles@gmail.com

(C) Education \& Psychology I+D+i and Ilustre Colegio Oficial de la Psicología de Andalucía Oriental (Spain) 


\section{Resumen}

Introducción. El objetivo principal de la investigación fue analizar la existencia de prejuicios en la población universitaria del Campus de Melilla (España) hacia los migrantes que cruzan la frontera en situación irregular. El papel de los educadores y el personal sanitario cobra un especial interés en este contexto, ya que son ellos los que van a tener un mayor contacto con los mismos. Esto hace necesario el desarrollo de investigaciones sobre esta temática.

Método. El estudio se centra en conocer si existen actitudes prejuiciosas en estudiantes universitarios, usando la Escala de prejuicio sutil y manifiesto hacia los migrantes en situación irregular en la Ciudad Autónoma de Melilla. La muestra está compuesta por 205 estudiantes de los Grados en Educación y Enfermería que se imparten en el Campus de Melilla. Se ha utilizado una metodología empírico-analítica siendo un estudio Ex Post Facto de tipo correlacional en el que se realizaron análisis descriptivos e inferenciales.

Resultados. Los resultados muestran que no existen diferencias significativas en torno a las actitudes prejuiciosas y la variable género. Por el contrario, los datos indican que la variable titulación y cultura de origen inciden en las actitudes que los universitarios muestran hacia las personas migrantes.

Discusión. Los resultados del estudio ponen de relieve la existencia de estudiantes universitarios con cierta carga de prejuicios hacia los migrantes en situación irregular. Preocupa que estudiantes de titulaciones de áreas tan sociales como la educación y la sanidad muestren este tipo de valoraciones hacia personas que, por diversos motivos, han tenido que emigrar de sus países (ya sea por pobreza, guerras, persecuciones políticas o por orientación sexual).

Palabras Clave: Prejuicio sutil; prejuicio manifiesto; actitudes; migrantes en situación irregular. 


\title{
Prejudiced attitudes of university students toward immigrants in an irregular situation: an exploratory study
}

\begin{abstract}
Introduction. The main objective of the research was to analyze the existence of prejudice among university students of Melilla Campus (Spain) towards migrants who cross the border illegally. The role of educators and health professionals has a special interest in this context; they are who will have more contact with them. This requires the development of research on this subject.

Method. The study focuses on knowing if there are prejudiced attitudes among university students, using the scale of subtle and blatant prejudice towards illegal immigrants in the Autonomous city of Melilla. The sample consists of 205 students. It has been used empiricalanalytical methodology to be an ex post facto correlational study in which descriptive and inferential analyzes were performed.
\end{abstract}

Results. The results show no significant differences regarding the prejudicial attitudes and gender variable. By contrast, the data indicates that the variable qualifications and culture of origin affect the attitudes which university students show about migrants.

Discussion and Conclusion. The results of the study highlight the existence of college students with certain amount of prejudice against irregular migrants. Concerned that students with social qualifications, such as primary education and nursing show this type of ratings to people who, for various reasons, have had to emigrate from their countries (either by poverty, wars, political persecution or sexual orientation).

Keywords: Subtle prejudice; blatant prejudice; attitudes; illegal migrants. 


\section{Introducción}

España vive en estos últimos años una presión migratoria espacial, siendo Melilla y Ceuta las dos puertas principales hacia Europa. La cantidad de personas que pasan de manera anual por nuestras fronteras es cada vez mayor. Entre los años 2005 y 2011 se produce un repunte en la entrada de migrantes en situación irregular, y una continua tendencia a la baja durante los siguientes años. A partir de 2013 las entradas aumentan hasta un 48,5\% entre las dos ciudades fronterizas (Europa Press, 2014) mientras que en 2014 la presión migratoria se ha disparado un 200\% (Gallego, 2014). Aumentando esta tendencia en 2015 (FRONTEX, 2015). La entrada masiva de migrantes de forma irregular provoca que el concepto de comunidad creado en la ciudad se distorsione, dando lugar al florecimiento o acentuación de rasgos racistas, xenófobos o prejuiciosos relacionados directamente con personas en esta situación. Estos términos hacen mención, de manera inevitable, a un concepto común a todos ellos, el de actitud.

\section{Actitudes hacia la inmigración}

A menudo en nuestra vida utilizamos el término actitud para referirnos a distintas cosas, sobre todo cuando hacemos referencia al comportamiento de las personas pero, ¿qué es la actitud? Desde la perspectiva de la psicología social el término ha ido evolucionando a lo largo de los años y adaptándose a los distintos paradigmas que han ido surgiendo, siendo muchas las definiciones que se han dado del concepto de actitud (Hovland, Janis, y Kelley, 1953; Hovland, 1959); desde la propuesta por Allport (1935) que la definía como "un estado de disposición mental y nerviosa, organizado mediante la experiencia, que ejerce un influjo directivo dinámico en la respuesta del individuo a toda clase de objetos y situaciones" (p. 810), hasta la planteada por Ovejero-Bernal (2010), que la definía como "una predisposición aprendida a responder de una manera conscientemente favorable o desfavorable respecto a un objeto dado, (físico, personas, grupos...)" (p.192) aunque se puede observar que en todas

ellas hay una serie de rasgos comunes, tales como: es un constructo no observable directamente (Ubillos, Páez, y Mayordomo, 2004); supone una predisposición a responder; se adquiere por aprendizaje y es perdurable en el tiempo (Worchel, Cooper, Goethals y Olson, 2002); presenta una estructura multidimensional organizada; $y$, formada por elementos cognitivos, afectivos y conativos (Rosenberg y Hovland, 1960). 
Las actitudes prejuiciosas del alumnado universitario hacia los inmigrantes

Por otro lado, son numerosos los modelos que a lo largo de los años han intentado explicar las dimensiones de las actitudes. Entre ellos destaca el modelo unidimensional de Petty y Cacioppo (1981) o el tridimensional, presentado por primera vez por Rosenberg y Hovland (1960). Actualmente, es este último uno de modelos más aceptados, también conocido como modelo ABC (Affective, Behavioral and Cognitive). Dicho modelo afirma que las actitudes están compuestas por tres componentes fundamentales: afectivo (conjunto de sensaciones o sentimientos que el objeto produce sobre el sujeto), cognitivo (conjunto de información que el sujeto posee sobre el objeto de actitud) y conductual (intenciones o tendencias propias del sujeto hacia un objeto actitudinal).

Cuando hablamos de actitudes, sobre todo racistas, hacia un exogrupo, no podemos evitar pensar en conceptos como el estereotipo, la discriminación o el prejuicio. Basándonos en las teorías básicas de Eagly y Chaiken (1993) y, siguiendo la propuesta multidimensional del modelo $\mathrm{ABC}$, se pueden relacionar cada uno de estos conceptos con cada una de las dimensiones propuesta; así pues, el modelo enmarca el estereotipo en la dimensión cognitiva (Stephan, 1989; Macrae, Stangor y Hewstone, 1996); la discriminación, en la conductual (Banaji y Gelman, 2013); y, los prejuicios en la afectiva (Devien, 1995; Stevens, 2016).

Los primeros, los estereotipos, como concepto, son introducidos en la psicología social por Lippmann (1922), que los definía como la imagen mental preconcebida que se activa en los individuos cuando piensa en un grupo social. La discriminación, y sus distintas formas como el racismo (moderno, simbólico, clásico), en cambio, según Dovidio, Hewstone, Glick, y Esses (2010) se definen como una conducta dirigida hacia miembros de un grupo, no como consecuencia de las acciones de éstos, sino, simplemente, por pertenecer a dicho grupo. Los prejuicios pueden ser considerados como actitudes negativas que se crean hacia otras personas o grupos. Para Light, Keller y Calhoun (1991) el prejuicio "es una predisposición categórica para aceptar o rechazar a las personas por sus características sociales reales o imaginarias” (p. 356). Así, es la década de 1920 el momento en la que se comienza a tener en cuenta el concepto de prejuicio pero es, a partir de los años 90, cuando surge un nueva perspectiva multidimensional del término junto con nuevas metodologías e instrumentos que permiten medirlo desde esta perspectiva (Cuadrado, 2007). Desde una perspectiva grupal, encontramos distintas teorías ampliamente aceptadas que estudian los determinantes del prejuicio, entre las que destacan: la teoría del contacto (Allport, 1954), la teoría del conflicto (Sherif, 1966, Le- 
vine y Campbell, 1971), la teoría de la identidad social (Tajfel, 1978) y la teoría de la congruencia de las creencias (Rokeach, 1960).

La primera de ellas, desarrollada por Allport (1954), sostiene que, uno de los factores que contribuye a la reducción de la hostilidad entre grupos, es el contacto entre los mismos, aunque el mero hecho de juntar distintos grupos en un mismo lugar no provoque por sí mismo esta reducción (Smith-Castro, 2011); la segunda, propuesta inicialmente por Sherif (1966), afirma que, si hay dos o más grupos en busca de un recurso común que escasea, surgirá un conflicto que d. Se ha utilizado una metodología empírico-analítica siendo un estudio Ex Post Facto de tipo correlacional en el que se realizaron análisis descriptivos e inferenciales. en actitudes de hostilidad, prejuiciosas o discriminatorias entre los mismos, creando así un ambiente de animadversión entre los distintos grupos que se ven inmersos en dicho conflicto; la tercera, desarrollada por Tajfel (1978), hace referencia al sentido de semejanza que percibe un sujeto respecto a otros que forman parte de su mismo grupo pero, además, se siente distinto con respecto a los miembros que forman parte de otros grupos (Morales y Yubero-Jiménez, 1999); y, la cuarta, propuesta por Rokeach (1960) afirma que, en los contextos en los que no existe presión social o no es eficaz, las creencias son el único medio disponible para que se produzca la discriminación racial o étnica.

Según Falanga, De Caroly Sagone (2014) y Fedor (2014), estereotipos y prejuicios están estrechamente relacionados, en la investigación de Gómez-Berrocal y Navas (2000) los sujetos más prejuiciosos presentan sentimientos más negativos hacia el exogrupo analizado; es por este motivo que introducimos una lista de adjetivos que buscan conocer cuáles son los rasgos característicos que los participantes les asignan a los inmigrantes en situación irregular, es decir, los estereotipos. Son Pettigrew y Meertens (1995), apoyándose en las teorías de Allport (1954), los primeros en demostrar que existen una diferenciación clara entre el prejuicio manifiesto y el sutil; el primero es la forma tradicional (vehemente, cercana y directa), mientras que el segundo es la forma moderna (frío, distante e indirecto). Estos mismos autores proponen una escala para la medición de dichos prejuicios que consta de dos subescalas, una para el prejuicio sutil y otra para el manifiesto. Estas subescalas, a su vez, se dividen en distintos factores (percepción de amenaza, oposición al contacto íntimo, defensa de los valores tradicionales, exageración de diferencias culturales y manifestación de emociones positivas) detectados en la investigación original de Pettigrew y Meertens (1995) y corroborados por Rueda y Navas (1996). 
Las actitudes prejuiciosas del alumnado universitario hacia los inmigrantes

Así, en la Escala Pettigrew y Meertens (1995) se establece tres grupos en los que se puede clasificar a la mayoría de sujetos. El primer grupo lo denominan igualitario (equalitarians) que se caracteriza porque puntúa bajo tanto en el prejuicio sutil como manifiesto; un segundo grupo, llamado sutiles (subtles) este grupo puntúa alto en la escala de prejuicio sutil y bajo en la de manifiesto y el tercer grupo es el denominado fanáticos (bigots), que obtiene alta puntuación tanto en la escala de prejuicio sutil como manifiesto. Los sujetos que puntúan bajo en prejuicio sutil y alto en manifiesto se consideran como erróneos y no válidos debido a que no cumplen con la estructura del constructo a medir (Augoustinos, Walker, y Donaghue, 2014). Aunque las investigaciones demostraban una disminución en las puntuaciones indicadoras de prejuicio en las escalas de actitudes, no sucedía lo mismo en los otros aspectos medidos, como la discriminación. Todo esto hacía ver que estos prejuicios en realidad no estaban disminuyendo, sino ocultándose o manifestándose de manera indirecta (Molero, Navas, y Cuadrado, 2006). Esto nos hace pensar que el concepto ha evolucionado y, aunque no se detecte de manera directa, podría darse de manera indirecta o sutil. Además, el contexto actual hace que sea un momento idóneo para analizar este tema en profundidad.

Actualmente en las sociedades occidentales nadie reconoce abiertamente ser prejuicioso hacia otros grupos por tener cultura, etnia, religión... diferente; pero hay que destacar las aportaciones de Morales y Moya (1996) que ante la pregunta ¿tendría usted reticencias en enviar a sus hijos a escuelas donde la mitad de los alumnos fueran negros?, el $80 \%$ de los encuestados en 1989 argumentó que no, mientras que, en 1942, sólo el 30\% de los encuestados habían defendido esta misma postura. Manifestar actitudes prejuiciosas no solo es poco deseable socialmente, sino que está incluso penado por la ley; por eso, entre otras razones, el prejuicio manifiesto ya no se expresa claramente. En esta línea, Montes (2008) manifiesta "la dicotomía entre el mantenimiento de actitudes prejuiciosas y el rechazo explícito a la expresión de las mismas, siendo poco deseable socialmente manifestar actitudes prejuiciosas en nuestra sociedad" (p. 6). Este ocultismo en cuanto a la consideración de las actitudes prejuiciosas lleva a diversos autores a plantearse la existencia de nuevos modelos de prejuicio, que es lo que denominan prejuicio sutil. Esta manifestación del prejuicio, no por ser sutil, es menos dañino para las personas objeto de él (Molero, Recio, García-Ael, Fuster y Sanjuán, 2012). 
La investigación de Navas, Cuadrado, Molero, y Alemán (2000) encontraron que los sujetos mostraban más prejuicio sutil hacia los migrantes subsaharianos y magrebíes siendo este último grupo el peor valorado. Además, concluyeron que estas nuevas actitudes prejuiciosas tienen como característica la ambivalencia, los sujetos apoyan políticas futuras de inmigración positivas, pero no están de acuerdo con la apertura de fronteras, y lo que es más significativo, cuando encuentran una justificación de tipo no racial defienden la expulsión controlada de los migrantes. Por otra parte, consideran que la llegada de migrantes ha traído consigo numerosas consecuencias negativas (aumento de los conflictos, delincuencia, etc.) pero a la vez, también reconocen su contribución al desarrollo económico de la zona y al enriquecimiento de nuestra cultura. Posteriormente, Navas, García, Rojas, Pumares y SánchezMiranda (2006) volvieron a constatar la supremacía de la expresión del prejuicio sutil sobre el manifiesto y hallaron que las puntuaciones más altas de prejuicio (manifiesto o sutil) se relacionan con un deseo de excluir (también de segregar en el caso del prejuicio afectivo) a ambos grupos de migrantes (magrebíes y subsaharianos), mientras que las puntuaciones más bajas están relacionadas con una preferencia por su integración.

Estas investigaciones no sólo se han centrado en las relaciones que tienen unos grupos con otros, sino que algunas han ido más allá intentando, por ejemplo, encontrar relaciones entre las puntuaciones de prejuicio sutil y manifiesto y la orientación política de los sujetos. A este respecto Gómez y Huici (1999) encuentran que las personas con afinidades políticas de derechas puntúan más alto en las formas sutiles que las de izquierdas. En el estudio de Guardia y Nacarí (2013) encontraron la existencia de un elevado número de sujetos que puntuaban más alto en la escala de prejuicio sutil que en la de manifiesto. En el mismo sentido, las personas que se identifican con ideas políticas de derecha manifiestan actitudes menos favorables hacia las minorías sociales en comparación con aquellas personas que se identifican con ideas de izquierda. Así mismo, las personas que manifiestan actitudes negativas hacia los exogrupos tienden a presentar posiciones contrarias hacia la ampliación de los derechos de éstos (Cárdenas, 2007; Cárdenas y Barrientos, 2008; Cárdenas, Music, Contreras y Calderón, 2007; Cea D'Ancona, 2009).

\section{Medida de las actitudes y prejuicios}

La manera en la que se miden las actitudes y los prejuicios se ha modificado en estos últimos años, sobre todo cuando tratamos temas relacionados con la inmigración (Etxeberria, Murua, Arrieta, Garmendia y Etxeberria, 2012). Se ha pasado de medir los prejuicios de una 
forma directa a una forma más sutil e indirecta, adaptándonos así a los cambios sociales producidos en la última década. Por otro lado, y siguiendo las investigaciones de Núñez-Alarcón, Moreno-Jiménez y Moral-Toranzo (2011) sobre la relación entre orientación religiosa y el prejuicio étnico concluyen que existe correlación entre religiosidad, en sus formas más conservadoras, y homofobia, sexismo e intolerancia religiosa, "siendo la Escala Prejuicio Religioso un instrumento útil para conocer la compleja relación religión-prejuicio" (p. 858). Sniderman y Tetlock (1986) analizaron la relación entre el prejuicio y escasez de recursos por parte de las sociedad (pobreza, crisis...) concluyendo que el prejuicio se hace más evidente en situaciones donde las tensiones entre los diferentes grupos sociales existentes influyen en la distribución de recursos económicos. Brown y Hewstone (2005) encuentran correlación entre el prejuicio étnico y racial con actitudes negativas hacia otras minorías. Así, las personas que manifiestan prejuicio contra minorías étnicas suelen obtener correlaciones altas con otras medidas de prejuicio general (Dunbar, 1995), sexismo (Glick y Fiske, 1995; King, 2003), prejuicio hacia las mujeres (Fiske y Von Herdy, 1992; Swim, Aikin, Hall y Hunter, 1995), hacia las personas homosexuales (Cárdenas, 2007) o referidas a antisemitismo (Dumbar, 1995; Dunbar y Simonova, 2003). Además, estas personas suelen mantener escaso contacto y bajos niveles de intimidad con las personas pertenecientes a los exogrupos. También se analiza la relación entre el sexo y el prejuicio étnico, concluyendo las investigaciones que las mujeres tienden a ser menos prejuiciosas que los hombres (Cárdenas y Barrientos, 2008a, 2008b) y que las personas más jóvenes y de nivel socioecómico más alto serían menos prejuiciosas que sus contrapartes (World Values Survey, 2005).

\section{Objetivos}

Hay que destacar que son pocas las investigaciones que se han desarrollado en un contexto tan singular como el de la Ciudad Autónoma de Melilla, la multiculturalidad presente en la ciudad y, el añadido de ser una de las fronteras sur de Europa, hacen que este tipo de estudios adquieren un valor extra y más aún cuando se desarrollan en un ámbito tan importante como es el universitario. Este estudio se centra en conocer si existen actitudes prejuiciosas en estudiantes universitarios hacia los migrantes en situación irregular en un contexto multicultural y en un ambiente en el que la inmigración parece ser un tema habitual en los últimos meses.

Por tanto, los objetivos de esta investigación son: por un lado, conocer el grado de prejuicio en el alumnado universitario hacia los migrantes en situación irregular y por otro, anali- 
zar las variables sociodemográficas que influyen en las actitudes prejuiciosas hacia este colectivo. En concreto, la variable sociodemográfica que suscitan un interés especial en el estudio es la cultura de origen de los participantes ya que la ciudad Autónoma de Melilla tiene como rasgo característico la convivencia entre diferentes culturas (Merino y Ruiz-Román, 2005; Sánchez-Fernández, 2010), siendo las dos religiones mayoritarias, la cristiana (cultura europea) y la musulmana (cultura bereber).

\section{Método}

\section{Participantes}

El total de participantes del estudio ha sido de 205 sujetos, 40 hombres (19,5\%) y 165 mujeres $(80,5 \%)$, con edades comprendidas entre 18 y los 28 años. Todos están cursando actualmente sus estudios en el Campus de Melilla, en la Facultad de Educación y Humanidades y la Facultad de Enfermería. En relación con las titulaciones analizadas se encuentran: los Grados en Educación Social (N=70), Educación Infantil (N=18), Educación Primaria ( $\mathrm{N}=32)$ y Enfermería ( $\mathrm{N}=50)$, y los Itinerarios Curriculares Concretos de Educación Primaria e Infantil ( $\mathrm{N}=35)$, siendo éstas las impartidas en las Facultades anteriormente citadas. En cuanto a la procedencia se agrupan en: 125 participante de procedencia europea, 71 alumnos de procedencia bereber, 2 judíos, 4 hindúes. El criterio de selección ha sido la titulación, seleccionando aquellas carreras en la que sus graduados tendrán mayor contacto con personas migrantes; por tanto, se ha utilizado un muestreo no probabilístico de tipo intencional.

\section{Instrumentos}

Escala de Prejuicio Sutil y Manifiesto (Pettigrew y Meertens, 1995). Validada tanto a nivel internacional, por los propios autores, como a nivel nacional por Rueda y Navas (1996). En esta escala, los participantes puntúan de 1 a 5 , siendo el rango de 1 "en total desacuerdo" hasta 5 "En total acuerdo". La escala cuenta de 20 ítems, 10 para prejuicio sutil y 10 para el prejuicio manifiesto. Estos mismos autores proponen dos subcategorías para el prejuicio manifiesto y tres para el sutil. Además, se añadió un apartado de datos sociodemográficos.

Aunque en la mayoría de investigaciones se emplea la media para separar los sujetos en distintas tipologías, se optó por un criterio más restrictivo, es decir, seleccionar aquellos sujetos que puntúan por encima del percentil 75, para evitar falsos positivos (ver tabla 1). Así encontramos que los fanáticos son quienes puntúan alto en ambas escalas, es decir, en la 
subescala sutil la puntuación es mayor o igual a 30 y en la escala manifiesta la puntuación es mayor o igual 27. En el caso de los sutiles, la puntuación es baja en manifiesto, pero alta en sutil, es decir, obtienen puntajes mayores o iguales a 30 en la escala sutil y en la escala manifiesta los valores obtenidos son menores o iguales a 27. Finalmente, los igualitarios son aquellos que puntúan bajo en ambas escalas, es decir, en la escala sutil la puntuación es menor a 30 y en la escala manifiesta es menor a 27. Algunos de los sujetos, por incongruencia con el constructo, fueron descartados ya que puntuaban en la escala sutil con valores menores a $30 \mathrm{y}$ en la escala manifiesta con puntuaciones mayores a 27 . Ver tabla 1.

Tabla 1. Cuartiles para el prejuicio sutil y manifiesto

\begin{tabular}{lccc}
\hline & & Sutil & Manifiesto \\
\hline $\mathrm{N}$ & & 205 & 205 \\
Media & & 25.4 & 22.3 \\
& 25 & 21 & 18 \\
Percentiles & 50 & 25 & 21 \\
& $\mathbf{7 5}$ & 30 & 27 \\
\hline
\end{tabular}

En cuanto a la fiabilidad de la Escala de Prejuicio Sutil y Manifiesto se realiza el Alfa de Cronbach obteniendo unos valores de $\alpha=.875$ para el prejuicio sutil, $\alpha=.802$ para el manifiesto y $\alpha=, 813$ para el total de las respuestas analizadas en conjunto. Estos datos demuestran la fiabilidad y consistencia interna del cuestionario utilizado. Aunque este estadístico ha sido ampliamente utilizado en la investigación social debemos completarlo con otro tipo de análisis para evitar sesgos propios de la prueba (Sijtsma, 2008), como los índices de fiabilidad compuesta y la varianza media extractada. Para calcular la fiabilidad compuesta (FC) y la varianza media extractada (VME) se llevó a cabo un análisis factorial confirmatorio. Los resultados obtenidos muestran para el prejuicio sutil una $\mathrm{FC}=.922$ y $\mathrm{VME}=.545 ; \mathrm{y}$, para el manifiesto, una $\mathrm{FC}=.908$ y $\mathrm{VME}=.504$. 
Listado de adjetivos positivos y negativos sobre emociones que despierta la persona inmigrante en situación irregular, extraídos de Gómez y Huici (1999). Contiene una lista de sentimientos y emociones puntuadas de 1 a 5, siendo 1 "nada", 2 "poco", 3 "algo", 4 "bastante" y 5 "mucho" y que se agrupan en positivas (atracción, simpatía y pena) y negativas (odio, hostilidad, inseguridad, miedo, envidia, incomodidad y asco), no estando obligados a responderlos todos.

\section{Procedimiento}

El procedimiento para la recogida de datos se efectuó de manera presencial siguiendo las instrucciones propias de los autores de las escalas. Se presentó a los estudiantes como una investigación para conocer su opinión acerca de los migrantes en situación irregular que estaban llegando a la Ciudad y que el cuestionario era totalmente anónimo y no estaban obligados a responderlo. El tiempo de aplicación fue, aproximadamente, entre 20 y 25 minutos. Se pidió permiso al docente responsable de la asignatura en la que se aplicó el cuestionario.

\section{Análisis de datos}

Para el análisis de los datos se utilizó el software de análisis estadísticos SPSS (Statistical Package for the Social Sciences) en su versión 22.0. Mediante el uso de dicho software se ha realizado estadísticos descriptivos y análisis de fiabilidad. Una vez contrastado el ajuste de la distribución normal de las puntuaciones, mediante la prueba de Kolmogorov-Smirnov con la corrección Lilliefors y Shapiro-Wilk; la homocedasticidad, mediante el estadístico de Levene; la independencia de los casos, mediante la prueba de rachas, se llevan a análisis estadísticos de contrastes ( $t$-Student, y ANOVA) para conocer la existencia o no de diferencias significativas entre los distintos grupos de participantes Para detectar la significatividad se ha utilizado un intervalo de confianza del $95 \%$.

\section{Resultados}

Atendiendo a puntos de corte obtenidos en la tabla 1 obtenemos los siguientes resultados en relación con las distintas variables analizadas (ver figuras 2 y 3). El 12.70\% se considera sujetos inválidados, el 60\% igualitarios, el $11.70 \%$ sutiles y el $12.70 \%$ fanáticos. 
Las actitudes prejuiciosas del alumnado universitario hacia los inmigrantes

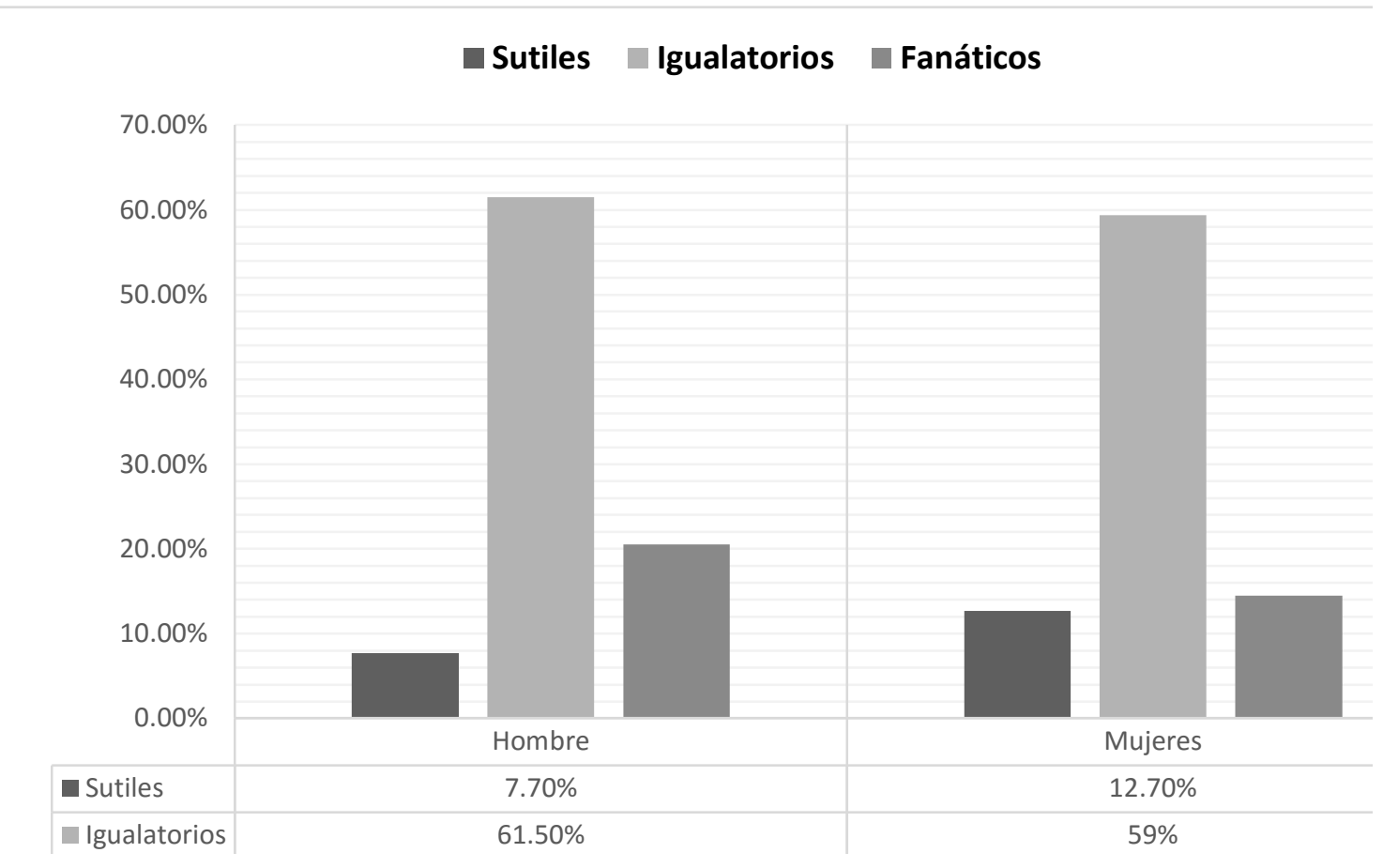

Figura 2. Tipología de participantes clasificados por sexo

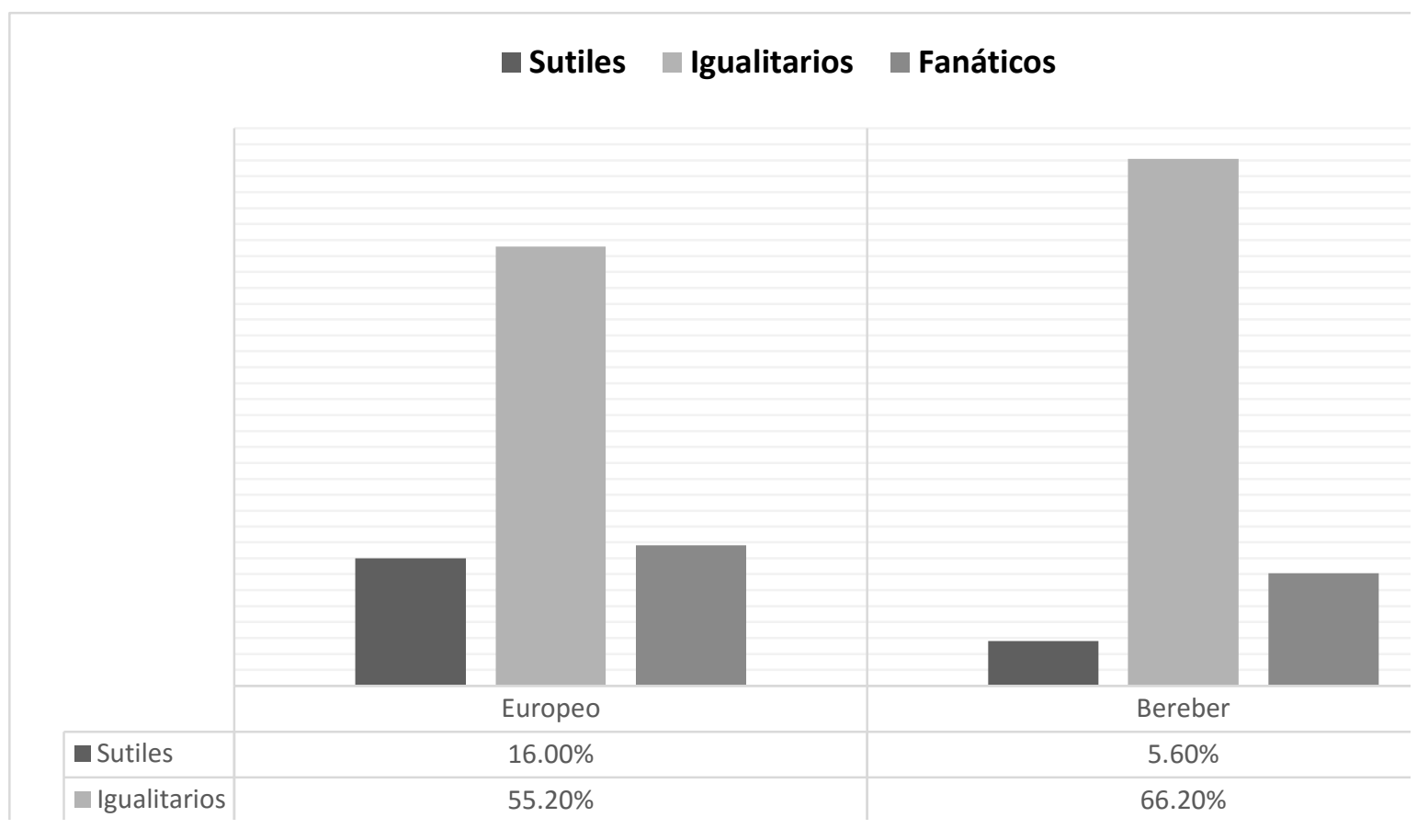

Figura 3. Tipología de participantes en base a la cultura de origen

La puntuación media de la escala de prejuicio manifiesto es de 27.17 (desviación típica 4.17 y mediana de 27) y la puntuación media de la escala de prejuicio sutil es de 28.52 (desviación típica 46 y mediana de 29). Los resultados señalan que la diferencia entre ambas 
puntuaciones es estadísticamente significativa $[t(205)=4.04 ; p=.001]$. Es decir, los sujetos con prejuicio sutil son mayores a los que presentan prejuicio manifiesto o tradicional. La correlación entre las puntuaciones de las dos escalas es estadísticamente significativa con un valor de $.570(p=.000)$.

Posteriormente se ha analizado si existen diferencias significativas en función de la cultura de origen de los universitarios. Para este análisis se atendió a las dos culturas mayoritarias que conforman la muestra, europea y bereber (conjunto de etnias autóctonas del norte de África, imazighen en lengua bereber). Los datos indican que existen diferencias en el prejuicio sutil pero no en el manifiesto. Son los de origen bereber los que presentan menor prejuicio sutil (Tabla 2).

Tabla 2. Prueba T de Student para Europeos y Bereberes

\begin{tabular}{ccccc}
\hline & Etnia & Media & $t$ & $p$ \\
\hline \multirow{2}{*}{ Sutil } & Europeo (125) & 29.18 & \multirow{2}{*}{2.215} & .028 \\
& Bereber (71) & 24.00 & & \\
\hline \multirow{2}{*}{ Manifiesto } & Europeo (125) & 22.71 & \multirow{2}{*}{1.272} & N.S. \\
& Bereber (71) & 21.36 & & \\
\hline
\end{tabular}

Por último, se ha realizado un análisis multivariado de varianza (MANOVA) tomando como factor la titulación a la que pertenecen, siendo las variables dependientes las puntaciones obtenidas tanto en prejuicio sutil como en manifiesto. Como medida del tamaño del efecto se ha utilizado el coeficiente eta cuadrado parcial $\left(\eta_{\mathrm{p}}{ }^{2}\right)$, ya que es uno de los procedimientos más utilizados habitualmente dentro de la investigación educativa (Sun, Pan, y Wang, 2010). Para la interpretación de los tamaños del efecto se utiliza el criterio establecido en el trabajo clásico de Cohen $(1988,1994)$ que sugiere el siguiente criterio orientativo: valores de entre 2 y .3 indican un efecto pequeño, alrededor de .5 un efecto mediano y mayores que .8 un efecto alto. Aunque en investigaciones en el área educativa, siguiendo a Morales (2012), un valor en torno a .30 se considera como relevante.

Una vez controlado el efecto de la variable titulación $[\lambda \mathrm{Wilks}=.741, \mathrm{~F}(7,885)=10 ; p$ $<.000, \eta_{\mathrm{p}}{ }^{2}=.139$; tamaño del efecto pequeño] los datos indican que hay un efecto de interacción estadísticamente significativo entre la variable independiente (la titulación de pertenen- 
Las actitudes prejuiciosas del alumnado universitario hacia los inmigrantes

cia) sobre las dependientes (los tipos de prejuicios que poseen). Teniendo en cuenta los datos referidos a cada variable dependiente considerada individualmente, hay diferencias estadísticamente significativas en función del tipo de prejuicio que poseen, obteniendo $\left[F(9.031)=17.94 ; p=.000, \eta_{\mathrm{p}}{ }^{2}=.156\right.$; tamaño del efecto pequeño] para el prejuicio sutil y $\left[F(8.453)=17.94 ; p=.000, \eta_{\mathrm{p}}{ }^{2}=.147\right.$; tamaño del efecto pequeño $]$ para el prejuicio manifiesto (ver tabla 3 y tabla 4 ).

Tabla 3. Medias de prejuicio sutil y manifiesto para cada titulación analizada.

\begin{tabular}{lcc}
\hline Titulación & Prejuicio Sutil & Prejuicio Manifiesto \\
\hline Educación Social & 22.41 & 19.84 \\
\hline Educación Infantil & 27.00 & 25.94 \\
\hline ICCs & 25.30 & 21.60 \\
\hline Enfermería & 28.14 & 22.06 \\
\hline Educación Primaria & 28.06 & 27.00 \\
\hline
\end{tabular}

Tabla 4. Resumen de la MANOVA para el factor titulación sobre las variables dependientes prejuicio sutil y prejuicio manifiesto.

\begin{tabular}{rccccccc}
\hline Fuente & Tipo prejuicio & M. & D.T. & $F$ & Sig. & $\eta_{p}{ }^{2}$ & $\begin{array}{c}\text { Potencia } \\
\text { Observada }\end{array}$ \\
\hline \multirow{2}{*}{ Titulación } & Sutil & 25.44 & 6.614 & 9.031 & .000 & .156 & .999 \\
\cline { 2 - 7 } & Manifiesto & 22.25 & 7.016 & 8.453 & .000 & .147 & .999 \\
\hline
\end{tabular}

* La diferencia de medias es significativa al nivel $\mathrm{p} \leq .05$

En la siguiente tabla se muestran las puntuaciones obtenidas tras realizar la prueba $U$ de Mann-Whitney ( $\mathrm{n}<30$ ) para detectar diferencias entre medias en los sujetos situados en los dos extremos, sutiles y fanáticos. Los resultados muestran la existencia de diferencias significativas en dos sentimientos concretos, la atracción y la pena. En el primero de ellos, la atracción, son los sutiles los que obtienen mayores puntajes en este sentimiento $\left(\mathbf{M}_{\text {sutil }}=2\right)$. En el segundo, la pena, son los sutiles quienes puntúan más alto $\left(\mathrm{M}_{\text {sutil }}=3.71\right)($ ver tabla 5$)$. 
Tabla 5. Prueba U de Mann-Whitney para los sentimientos según la tendencia sutil o manifiesta de los sujetos

\begin{tabular}{ccccc}
\hline & Sutil $(\mathrm{M})$ & Fanático $(\mathrm{M})$ & $\mathrm{U}$ & $p<$. \\
\hline Odio & 1.13 & 1.30 & 296.5 & .182 \\
\hline Atracción & 2 & 1.23 & 171 & .001 \\
\hline Hostilidad & 1.64 & 1.83 & 266 & .198 \\
\hline Miedo & 2.04 & 2.56 & 277.5 & .067 \\
\hline Envidia & 1.04 & 1 & 330 & .253 \\
\hline Simpatía & 2.71 & 2.32 & 258 & .192 \\
\hline Incomodidad & 2.18 & 2.42 & 296 & .399 \\
\hline Asco & 1.27 & 1.68 & 298 & .345 \\
\hline Pena & 3.71 & 2.94 & 251.5 & .034 \\
\hline Inseguridad & 2.63 & 2.84 & 355.5 & .628 \\
\hline
\end{tabular}

\section{Discusión y Conclusiones}

Al comparar los resultados obtenidos podemos afirmar que están acordes con los estudios originales y las distintas adaptaciones que se han hecho en distintos países (Pettigrew y Meertens, 1995; Navas, Cuadrado, Molero, y Alemán, 2000; Cárdenas, Music, Contreras, Yeomans, y Calderón, 2007).

Atendiendo a la tipología de los sujetos se ha encontrado que la mayor parte de los mismos, según la propuesta por Pettigrew y Meertens (1995), se clasifican en igualitarios $(60 \%)$. Se observa que el porcentaje de sujetos fanáticoses es mayor a los sutiles. Esto indica que a los participantes no les importa manifestar sus creencias prejuiciosas de forma abierta hacia los inmigrantes irregulares, aunque la cantidad en relación a la muestra no es destacable. Estos resultados no coinciden con la investigación de Espelt, Javaloy y Cornejo (2006), en las que el grupo de fanáticos es significativamente menor que el de sutiles. Por el contrario, los resultados obtenidos están en la línea de Molero, Navas y Cuadrado (2006) en las que el exogrupo objeto de estudio es comparable al que se encuentra en la ciudad de Melilla. Este resultado es preocupante ya que el ámbito profesional de los participantes (Educación y Sanidad) son los que mantienen un contacto más directo con los migrantes. No obstante, este dato 
Las actitudes prejuiciosas del alumnado universitario hacia los inmigrantes

contrasta con la obtención de un $60 \%$ de estudiantes que se encuentran en el grupo de igualitarios, por tanto, con bajos niveles de prejuicios.

Comparando nuestra investigación con el estudio de Guardia y Nacarí (2013) se han obtenido unos resultados distintos. Estos autores han obtenido un elevado número de sujetos que puntúan más alto en la escala de prejuicio sutil que en la de manifiesto. No obstante, y a pesar de ser un estudio realizado con estudiantes universitarios, el contexto social en el que se ha desarrollado el estudio difiere enormemente con esta investigación. En Melilla, el contacto con personas migrantes en situación irregular y la presión migratoria es muy alta.

Tras realizar la prueba $t$ de Student para las puntuaciones de prejuicio sutil y manifiesto se ha encontrado que solo existen diferencias significativas para el prejuicio sutil, siendo los de origen europeo los que muestran mayores actitudes prejuiciosas. Una posible explicación a este resultado puede deberse, basándonos en la teoría del contacto de Allport (1954), a que el grupo cultural de origen bereber puede sentirse identificado, de alguna manera, con los migrantes por cuestiones de índole religiosa, cultural, etc. mientras que, como afirman Licata y Klein (2002), la identificación de los sujetos como europeos de pleno derecho provoca una menor tolerancia hacia los extranjeros. Además, no se han encontrado investigaciones que relacionen directamente la cultura de origen europea y bereber con este tipo de prejuicios.

Al analizar la muestra en relación a la titulación a la que pertenecen los sujetos, los datos indican que son los estudiantes del Grado en Educación Social los que presentan menores actitudes prejuiciosas, tanto sutiles como manifiestas hacia los migrantes, frente a los estudiantes de las otras titulaciones. En esto puede indicar que las características de los estudiantes que acceden a estas titulaciones difieren tanto en la predisposición favorable hacia este colectivo como en su compromiso social, ya que el alumnado de educación social suele presentar un alto compromiso con los grupos más desfavorecidos.

En relación con la tipología y los sentimientos y sus medias obtenemos que las puntuaciones en pena y simpatía son las más altas, incluso en el grupo considerado fanático. Por otro lado, tanto en el grupo de sutil como en el igualitario destaca un adjetivo con connotaciones negativas, la inseguridad. Por tanto, los sujetos con perfiles sutil y manifiesto muestran sentimientos negativos hacia los migrantes en situación irregular, estos resultados están acordes con el estudio de Molero, Navas y Cuadrado (2006) sobre las nuevas teorías del prejuicio, que estas-defienden la poca evocación de sentimientos, tanto positivos como negativos, hacia los migrantes. Por el contrario, en la investigación realizada por García, Navas, Cuadrado y 
Molero (2003), los resultados indican que tanto las emociones negativas (odio, hostilidad, rabia y asco) como las positivas (atracción y simpatía) predicen el racismo moderno hacia el exogrupo.

Los resultados del estudio ponen de relieve la existencia de estudiantes universitarios con cierta carga de prejuicios hacia los migrantes en situación irregular. Preocupa que estudiantes de titulaciones de áreas tan sociales como la Educación y la Sanidad muestren este tipo de valoraciones hacia personas que, por diversos motivos, han tenido que migrar de sus países (ya sea por pobreza, guerras, persecuciones políticas o por orientación sexual), sobre todo porque, la existencia de cualquier tipo de prejuicio, puede desembocar en discriminación, violencia o acoso, sobre todo cuando el exogrupo es considerado como inmigrante (McDuie-Ra, 2012).

En esta investigación se muestra, al igual que en los trabajos de Espelt, Javaloy y Cornejo (2006), Pérez (1996), Pettigrew y Meertens (1995), Rueda y Navas (1996) y Khalfani (2006), que la manifestación de los prejuicios hacia este tipo de personas ha ido transformándose hacia formas más encubiertas (prejuicio sutil) y que generan menos rechazo social.

Por tanto, podemos afirmar que, dando respuesta a nuestro objetivo de investigación, existen estudiantes del Campus de Melilla (España) presentan prejuicios hacia los migrantes en situación irregular, y que se precisa, por tanto, de una intervención orientada hacia la eliminación de estas actitudes, máxime cuando el componente social de estas titulaciones es tan alto.

\section{Limitaciones y propectivas}

Las limitaciones del estudio se centran principalmente en el bajo número de participantes, viéndonos restringidos por la población universitaria actual de la Ciudad. Como posibles propuestas de mejora para la investigación se proponen las siguientes: aumentar la muestra de participantes y extenderla a la totalidad del campus; analizar estas mismas relaciones en una ciudad parecida en contexto a Melilla, como Ceuta; analizar las relaciones entre los endogrupos y con otros exogrupos culturales. 
Las actitudes prejuiciosas del alumnado universitario hacia los inmigrantes

\section{Referencias}

Allport, G. W. (1935). Attitudes. En C. M. Murchison (Ed.), Handbook of Social Psychology. Winchester, MA: Clark University Press

Allport, G. W. (1954). The nature of prejudice. Reading: Addison-Wesley.

Augoustinos, M., Walker, I., y Donaghue, N. (2014). Social Cognition: An Integrated Introduction. Singapur: Sage.

Banaji, M. y Gelman, S. (2013). Navigating the social world. Oxford: Oxford University Press.

Brown, R., y Hewstone, H. (2005). An integrative theory of intergroup contact. En M. Zanna (Ed.), Advances in experimental social psychology (Vol. 37, pp. 255-343). San Diego, CA: Academic Press.

Cárdenas, M. (2007). Escala de racismo moderno: Propiedades psicométricas y su relación con variables psicosociales. Universitas Psychologica, 6 (2), 255-262.

Cárdenas, M. y Barrientos, J. (2008a). The attitudes toward lesbians and gay men scale (ATLG): Adaptation and testing the reliability and validity in Chile. Journal of Sex Research, 45(2), 140-149. Doi: 0.4067/s0718-22282008000200002

Cárdenas, M. y Barrientos, J. (2008b). Actitudes explícitas e implícitas hacia los hombres homosexuales en una muestra de estudiantes universitarios en chile. Psykhe, 17 (2), 17 25. Doi: 10.1080/00224490801987424

Cárdenas, M., Music, A., Contreras, P., Yeomans, H., y Calderón, C. (2007). Las nuevas formas de prejuicio y sus instrumentos de medida. Revista de Psicología, 7 (1) 69-96. Doi: 10.5354/0719-0581.2007.18435

Cea D’Ancona, M. A. (2009). La compleja detección del racismo y la xenofobia a través de encuesta. Un paso adelante en su medición. Revista Española de Investigaciones Sociológicas, 125, 13-45.

Cohen, J. (1988). Statistical power analysis for the behavioral sciences ( $2^{\mathrm{a}}$ ed.). Hillsdale, NJ: Erlbaum.

Cohen, J. (1994). The earth is round ( $\mathrm{p}<.05)$. American Psychologist, 49, 997-1003. Doi: 10.1037/0003-066x.49.12.997

Connolly, P., Kelly, B., y Smith, A. (2009). Ethnic habitus and young children: a case study of Northern Ireland. European Early Childhood Education, 2 (17), 217-232. Doi: $10.1080 / 13502930902951460$ 
Cristol, D., y Gimbert, B. (2008). Racial Perceptions of Young Children: A Review of Literature Post-1999. Early Childhood Education Journal, 36 (2), 201-207. Doi: $10.1007 / \mathrm{s} 10643-008-0251-6$

Cuadrado, I. (2007). Psicología social del prejuicio y la aculturación. En I. Cuadrado e I. Fernández (Eds.), Psicología Social (pp. 133-163). Madrid: Sanz y Torres.

Devine, P. G. (1995). Prejudice and out-group perception. En A. Tesser (Ed.), Advanced Social Psychology (pp.467-524). Nueva York: McGraw-Hill.

Dunbar, E. y Simonova, L. (2003). Individual difference and social status predictors of antisemitism and racism US and Czech findings with the prejudice/tolerance and right wing authoritarianism scales. International Journal of Intercultural Relations, 27, 507-523. Doi: 10.1016/s0147-1767(03)00051-8

Dunbar, E. (1995). The Prejudiced Personality, Racism, and Anti-Semitism: The PR Scale Forty Years Later. Journal of Personality Assessment, 65 (2), 270-277. Doi: 0.1207/s15327752jpa6502_4

Dovidio, J., Hewstone, M., Glick, P., y Esses, V. (2010). The SAGE Handbook of Prejudice, Stereotyping and Discrimination. London: Sage Publications.

Eagly, A. H., y Chaiken, S. (1993). The psychology of attitudes. Fort Worth: Harcourt Brace Jovanovich College Publishers.

Espelt, E., Javaloy, F., y Cornejo, J. M. (2006). Las escalas de prejuicio manifiesto y sutil: ¿una o dos dimensiones? Anales de Psicología, 22 (1), 82-88.

Etxeberria, F., Murua, H., Arrieta, E., Garmendia, J., y Etxeberria, J. (2012). Prejuicios, inmigración y educación. Actitudes del alumnado de secundaria. Revista Interuniversitaria De Formación Del Profesorado, 26 (3), 97-131.

Europa Press. (2014). La entrada ilegal de inmigrantes en Ceuta y Melilla repunta y aumenta un 49\% en 2013. El Mundo. Recuperado el 14 de abril 2014 de http://www.elmundo.es/espana/2014/04/29/535fbbc0ca47419e1f8b4573.html

Falanga, R., De Caroli, M., y Sagone, E. (2014). The Relationship between Stereotypes and Prejudice toward the Africans in Italian University Students. Procedia - Social and Behavioral Sciences, 159, 759-764. Doi: 10.1016/j.sbspro.2014.12.444

Fedor, C. (2014). Stereotypes and Prejudice in the Perception of the "Other". Procedia - Social and Behavioral Sciences, 149, 321-326. Doi: 10.1016/j.sbspro.2014.08.257

Fiske, S. y Glick, P. (1995). Ambivalence and Stereotypes Cause Sexual Harassment: A Theory with Implications for Organizational Change. Journal of Social Issues, 51 (1), pp.97-115. Doi: 10.1111/j.1540-4560.1995.tb01311.x 
Fiske, S. y von Hendy, H. (1992). Personality feedback and situational norms can control stereotyping processes. Journal of Personality and Social Psychology, 62 (4), pp.577596. Doi: 10.1037/0022-3514.62.4.577

FRONTEX. (2015). FRAN Quarterly 1. Warsaw (Poland): European Agency for the Management of Operational Cooperation at the External Borders of the Member States of the European Union.

Gallego, J. (2014, mayo). La presión migratoria en Ceuta y Melilla se dispara un 200\%. El Mundo. Recuperado el 01 de abril 2015 de http://www.elmundo.es/espana/2014/05/14/53735075e2704e42388b4573.html

García, M., Navas, M., Cuadrado, M., y Molero, F. (2003). Inmigración y prejuicio: actitudes de una muestra de adolescentes almerienses. Acción Psicológica, 2 (2). Doi:10.5944/ap.2.2.527

Gómez, Á., y Huici, C. (1999). Orientación política y racismo sutil y manifiesto: relaciones con la discriminación. Revista de Psicología Social: International Journal of Social Psychology, 14 (3), 159-180. Doi: 10.1174/021347499760259921

Gómez-Berrocal, C. y Navas, M. (2000). Predictores del prejuicio manifiesto y sutil hacia los gitanos. Revista De Psicología Social, 15 (1), 3-30. Doi: $10.1174 / 021347400760259848$

Guardia, L., y Nacarí, G. (2013). Discriminación racial dentro del ámbito universitario. Un enfoque sobre la discriminación sutil y manifiesta. Revista electrónica de investigación Docencia Creativa, 2, 111-116.

Herrero, J., Rodríguez, F. J., y Musitu, G. (2014). Comparing intergroup contact effects on blatant and subtle prejudice. Psicothema, 26 (1), 33-38. Doi: $10.7334 /$ psicothema2013.163

Hovland, C., Janis, I., y Kelley, H. (1953). Communication and persuasion. New Haven: Yale University Press.

Hovland, C. (1959). Reconciling conflicting results derived from experimental and survey studies of attitude change. American Psychologist, 14 (1), 8-17. Doi: $10.1037 / \mathrm{h} 0042210$

Khalfani, A. (2006). The hidden debate. New York: Routledge.

King, K. (2003). Racism or Sexism? Attributional ambiguity and simultaneous membership in multiple oppressed groups. Journal of Applied Social Psychology, 33 (2), 223-247. Doi: 10.1111/j.1559-1816.2003.tb01894.x 
LeVine, R., y Campbell, D. (1971). Ethnocentrism: theories of conflict, ethnic attitudes, and group behavior. New York: Wiley.

Light, D., Keller, S., y Calhoun, C. (1991). Aproximaciones a la Sociología. México: McGrawn-Hill.

Licata, L. y Klein, O. (2002). Does European citizenship breed xenophobia? European identification as a predictor of intolerance towards immigrants. Journal of Community \& Applied Social Psychology, 12 (5), 323-337. Doi: 10.1002/casp.684

Lippmann, W. (1922). Public Opinion. New York: Harcourt, Brace.

McDuie-Ra, D. (2012). Northeast migrants in Delhi. Amsterdam: Amsterdam University Press.

Macrae, C., Stangor, C., y Hewstone, M. (1996). Stereotypes and stereotyping. New York: Guilford Press.

Merino, D. y Ruiz-Román, C. (2005). Actitudes de los profesores hacia la educación intercultural. Aula Abierta, (86), 185-204.

Molero, F., Navas, M. S., y Cuadrado, M. I. (2006). Las nuevas expresiones del prejuicio racial: aspectos teóricos y empíricos. En C. Huici Casal, y F. J. Morales Domínguez, Estudios de psicología social (Coords.) (pp. 85-120). Madrid: Universidad Nacional de Educación a Distancia.

Molero, F., Recio, P., García-Ael, C., Fuster, M., y Sanjuán, P. (2012). Measuring Dimensions of Perceived Discrimination in Five Stigmatized Groups. Social Indicators Reseach, 114 (3), 901-914. Doi: 10.1007/s11205-012-0179-5

Montes, B. (2008). Discriminación, prejuicio, estereotipos: conceptos fundamentales, historia de su estudio y el sexismo como nueva forma de prejuicio. Iniciación a la investigación, 1 (3), 1-16.

Morales, J. F., y Moya, M. C. (1996). Tratado de Psicología Social. Vol. I: Procesos básicos. Madrid: Síntesis.

Morales, J. y Yubero-Jiménez, S. (1999). El grupo y sus conflictos. Cuenca: Servicio de Publicaciones de la Universidad de Castilla-La Mancha.

Navas, M., Cuadrado, I., Molero, F., y Alemán, P. (2000). Una aproximación psicosocial a la inmigración africana en un municipio del poniente almeriense: causas, consecuencias y políticas futuras. En actas II Congreso sobre la Inmigración en España "España y las Migraciones Internacionales en el cambio de siglo" (pp. 1-16). Madrid. 
Las actitudes prejuiciosas del alumnado universitario hacia los inmigrantes

Navas, M., García, M., Rojas, A., Pumares, P., y Sánchez-Miranda, J. (2006). Actitudes de aculturación y prejuicio: un estudio desde la perspectiva de autóctonos e inmigrantes en Almería. Psicothema, 18 (2), 187-193.

Nesdale, D. (2001). Language and the development of children's ethnic prejudice. Journal of Language And Social Psychology, 20 (1), 90-110. Doi: 10.1177/0261927X01020001005

Núñez-Alarcón, M., Moreno-Jiménez, P. y Moral-Toranzo (2011). Modelo causal del prejuicio religioso. Anales de Psicología, 27 (3), 852-861.

Pérez, J. A. (1996). Nuevas formas de racismo. En J.F. Morales y S. Yubero (Eds.), Del prejuicio al racismo: perspectivas psicosociales (pp. 79-102). Cuenca: Ediciones de la Universidad de Castilla La mancha.

Ovejero-Bernal, A. (2010). Psicología social. Madrid: Biblioteca Nueva.

Pettigrew, T., y Meertens, R. (1995). Subtle and blatant prejudice in Western Europe. European Journal of Social Psychology, 1 (25), 57-75. doi:10.1002/ejsp.2420250106

Petty, R., y Cacioppo, J. (1981). Attitudes and persuasion: classic and contemporary approaches. Dubuque: WIlliam C. Brown.

Rokeach, M. (1960). The open and closed mind. New York: Basic Books.

Rosenberg, M., y Hovland, C. (1960). Cognitive, Affective and Behavioral Components of Attitudes. En M. Rosenberg, y C. Hovland, Attitude Organization and Change: An Analysis of Consistency Among Attitude Components (pp. 1-14). New Haven: Yale University Press.

Rueda, J., y Navas, M. (1996). Hacia una evaluación de las nuevas formas de prejuicio racial: las actitudes sutiles del racismo. Revista de Psicología Social, 2 (11), 131-149. Doi: $10.1174 / 02134749660569314$

Sánchez Fernández, S. (2009). Convivencia escolar y diversidad cultural. Madrid: Secretaría General Técnica del Ministerio de Educación, Subdirección General de Documentación y Publicaciones.

Sherif, M. (1966). Common predicament: Social psychology of intergroup conflict and cooperation. Boston: Houghton Mifflin

Sijtsma, K. (2008). Reliability Beyond Theory and Into Practice. Psychometrika, 74 (1), 169173. http://dx.doi.org/10.1007/s11336-008-9103-y

Smith-Castro, V. (2011). La psicología social de las relaciones intergrupales: modelos e hipótesis. Actualidades en psicología, 20 (107), 45. Doi:10.15517/ap.v20i107.37 
Sniderman, P. y Tetlock, P. (1986). Reflections on American Racism. Journal of Social Issues, 42 (2), pp.173-187. Doi: 10.1111/j.1540-4560.1986.tb00231.x

Stephan, W. (1989). A Cognitive Approach to Stereotyping. En D. Bar-Tal, C. Graumann, A. Kruglanski \& W. Stroebe, Stereotyping and Prejudice: Changing Conceptions ( $1^{\mathrm{a}} \mathrm{ed}$., pp. 37-59). Nueva York: Springer Science Business Media.

Stevens, P. (2016). Ethnicity and racism in Cyprus. Hampshire (England): Palgrave Macmillan UK.

Sun, S., Pan, W., y Wang, L. L. (2010, September 20). A Comprehensive Review of Effect Size Reporting and Interpreting Practices in Academic Journals in Education and Psychology. Journal of Educational Psychology. Advance online publication. doi: $10.1037 / \mathrm{a} 001950$

Swim, J., Aikin, K., Hall, W. and Hunter, B. (1995). Sexism and racism: Old-fashioned and modern prejudices. Journal of Personality and Social Psychology, 68 (2). Doi: 10.1037/0022-3514.68.2.199

Tajfel, H. (1978). Differentiation between social groups. London: Academic Press.

Ubillos, S., Páez, D., y Mayordomo, S. (2004). Actitudes: definición y medición: Componentes de la actitud. Modelo de acción razonada y acción planificada. En I. FernándezSedano, S. Ubillos, E. Zubieta y D. Páez, Psicología social, cultura y educación ( $1^{\mathrm{a}}$ ed., pp. 301-326). Madrid (España): Pearson Educación.

Worchel, S., Cooper, J., Goethals, G. R. y Olson, J. M. (2002). Psicología social. México: Thompson.

World Values Survey (2005). World Values Survey. Stockholm. Suecia. Extraído el 20 de agosto de 2010, de http://www.worldvaluessurvey.org/wvs.jsp 